\title{
TOWARDS A CLOUD BASED SME DATA ADAPTER FOR SIMULATION MODELLING
}

\author{
James Byrne \\ PJ Byrne \\ Diana Carvalho e Ferreira \\ Anne Marie Ivers \\ Dublin City University Business School \\ Dublin City University \\ Dublin 9, IRELAND
}

\begin{abstract}
Discrete event simulation (DES) is a technique used extensively and effectively by large companies, however it is not widely used by small to medium sized enterprises (SMEs) due to complexity and related costs being prohibitively high. In SMEs, DES-related data can be stored in a variety of formats and it is not always evident what data is required (if even available) to support a DES model in relation to specific problem scenarios. Therefore the DES data gathering and preparation phase is where complexity and effort required are highest in order to avoid the potential for erroneous results due to incorrect assumed or real input data. The proposed solution is a Cloud-based adapter that can identify and connect to existing data sources and/or fills gaps in data in relation to defined problem scenarios, thus lowering the barriers for SMEs to gain benefit from DES studies due to reduced complexity and effort.
\end{abstract}

\section{INTRODUCTION}

A Discrete Event Simulation (DES) model is a computer based surrogate for experimenting with a real system, which is often infeasible or not cost-effective. DES models primarily consist of entities (e.g. products, customers), which have attributes (e.g. product/customer type), which require processing resources (e.g. machines, employees) and which closely mimic characteristics of a real-life process or systems (e.g. operational logic), such as randomness or variability (e.g. machine breakdowns, demand changes). While the use of DES is prevalent in large organizations, within small to medium sized enterprises (SMEs) it is not widely used. Within large organizations, DES is extensively used in situations where real systems cannot be used for experimentation. It is widely used in a vast array of industrial sectors right across the world. As a technique, DES has three main phases: data gathering and preparation, model development and model experimentation. The first phase (data gathering and preparation) can be both costly and time consuming due to data being stored in a variety of formats, difficulties in identifying what data is required to solve a specific problem scenario, and data not always being available or stored. This phase is the least supported of these three phases. Indeed for models developed specifically for SMEs, there is little evidence in literature of support for this phase in existence.

In this work the focus is on the first phase, namely data gathering and preparation for DES model development in SMEs. Within large organizations, data population of DES models (although complex and iterative) is generally completed through automatic connectivity links between organizational databases and/or manual upload/input to embedded model data tables. Data feeds to DES models originate from a wide variety of sources as dictated by the particular scenario under review. Examples of data sources range from manufacturing execution systems (MES), enterprise resource planning (ERP) systems, human resource (HR) employee databases, customer relationship management (CRM) tools, supplier relationship management (SRM) tools right through to localized spreadsheets. 


\section{Byrne, Byrne, Carvalho e Ferreira, and Ivers}

Large companies generally have larger budgets available to support DES studies, and the required data is typically stored in a more structured way, and is typically more readily available. However, at an SME level, budgets are not always readily available, and in order to perform a DES study time can be taken up in the complexity involved with data identification in relation to the specific problem to be solved, data gathering from a variety of sources and (where data is not available) data generation. This complexity is a barrier to DES adoption by SMEs due to higher costs in relation to time. This research focuses on reducing this cost and complexity through introducing a proposed solution which enables a reduction in the time and an increase the accuracy necessary to complete the DES data gathering and preparation phase for specific problem scenarios. In this way, data can be presented in a DES ready format reducing the barriers for DES adoption by SMEs.

\section{BACKGROUND}

Enterprises are under pressure to become more responsive and agile and to improve their performance to gain or maintain a competitive advantage (Simchi-Levi, Kaminsky, and Simchi-Levi 2003). Key competitive advantages for manufacturing enterprises include responsiveness and agility. DES is one method used by enterprises in order to increase performance and become more competitive. There are a number of ways that DES can help accomplish this: DES can show how processes will operate when process influencing variables are introduced, modified or withdrawn; it has been proven as a reliable decision support tool that can contribute to the process of continuous improvement; it can provide a platform to support the analysis and evaluation of "what if" scenarios; it can identify bottlenecks that result in high inventory levels and low resource and machine utilization (O'Kane, Papadoukakis, and Hunter 2007).

Small to medium-sized enterprises (SMEs) are of crucial importance to most economies worldwide. Although there is no single generalized definition for an SME, they are most widely seen as companies typically with less than 500 employees in the USA (U.S. Small Business Administration 2013), or in Europe with less than 250 employees and a turnover of less than $€ 50$ million or a balance sheet total of less than $€ 43$ million (European Commission 2003). SMEs are also seen as principal drivers for innovativeness and economic growth. Other than size, Casson (1982) documented that uncertainty is the central characteristic which distinguishes small from large firms. According to Koh and Saad (2002), uncertainty can be defined as unpredictable events in manufacturing environments that disturb the operations and performance of an enterprise. Small firms are often vulnerable in that they are more likely to have a single or a limited range of products/services so that they are more exposed by changes in the marketplace.

Outside of isolated one-off studies, the role of DES within SMEs has not generally received much attention by researchers. It has been somewhat overlooked and most simulations tend to focus on larger organizations due to the prohibitive cost of DES tools and the expertise required to develop credible models, however there is a need for SMEs to improve their production processes and to apply DES tools particularly aimed at the operational aspects of their business (O'Kane, Papadoukakis, and Hunter 2007). DES models can provide a unique insight into production issues and problems within the SME sector and models can help to identify opportunities for system improvements. In particular, production improvement in terms of production planning, scheduling, layout planning and optimization of processes are key areas in which DES can support operational decision-making (O'Kane, Papadoukakis, and Hunter 2007).

The lifecycle of a DES study goes through a number of phases, (Law 2009, Balci 1994, Balci 1997). The three main DES phases can be described as "data gathering and preparation", "model development" and "model experimentation". The first phase, data gathering and preparation, involves identifying, understanding, gathering and validating data for use in the DES model. The second phase, model development, covers problem formulation, defining objectives, conceptual modeling and programming the DES model. The third phase, model experimentation, includes DES experimentation and analysis of both the DES runs and the results obtained. The first phase can be both costly and time consuming and out of the three phases is the least supported phase. Indeed for models developed specifically for small to medium sized enterprises (SMEs), there is little evidence of support for this phase in existence. 


\section{Byrne, Byrne, Carvalho e Ferreira, and Ivers}

In general, some efforts around the use of standardizing the exchange of data have been made. For example, efforts have been made towards the development of mechanisms for the efficient exchange of information and data between simulations and other manufacturing tools. The Core Manufacturing Simulation Data (CMSD) standard is under development to address these issues whereby neutral representations for the core manufacturing entities that need to be exchanged between simulations and other applications are developed using a Unified Modeling Language (UML) information model, (Riddick and Lee 2008; Leong, Lee, and Riddick 2006; Bergmann, Stelzer, and Straßburger 2011). The potential use of SysML for DES has also been studied, (Liston et al. 2010; Huang, Ramamurthy, and McGinnis 2007). In addition, Bengtsson et al. (2009) describe and implement a methodology to address input data management in DES projects through the use of a generic data management tool which can extract, process, structure and present input data in CMSD format. However, these efforts, while potentially applicable, have not been focused at an SME level, in particular they do not address the issues of identification of data in relation to specific problem scenarios, or aiding in data generation where data is not readily available. The next section gives an overview of requirements for a data adapter for SMEs in the manufacturing space through initial SME interaction and focused group sessions.

\section{SME DATA ADAPTER REQUIREMENTS}

Within SMEs data can be stored in a variety of formats, ranging from paper-based (hard-copy data) through to data persistence on the Cloud. As part of the requirements engineering process, an initial study took place with four manufacturing SMEs in order to elicit, analyze and specify requirements for a data adapter tool for SMEs in addition to understanding the needs, usefulness and potential for DES in different SME sectors. A summary of the results of this preliminary effort can be seen in Table 1. Referring to this table, Company 1 is a manufacturing SME involved in producing food products. They have between 51-100 employees and they typically deal with manufacturing products with low volumes and high variety. Their method of storing data is primarily through recording data in a manual way through the use of paper based forms, stored on-site. In this case, software is not used extensively, and they require the use of DES in order to understand their system to enable improvements in terms of the effect on capacity with fluctuating demand. Therefore through the high level requirements elicitation process (conversational and structured interviews), it became evident that a tool in this space would be required to have the ability to capture manual paper-based data as well as the ability to capture human-based systems knowledge.

Company 2 is a manufacturing SME in the medical sector with between 201 and 300 employees. Their production process is highly automated in which un-scheduled downtime of machines and changeovers can be significant, leading to an established potential need for DES modeling capability in order to aid them in terms of adherence to schedules. Through the requirements elicitation process, it was established that the data relevant to the required system to be modeled is stored in an ERP system, with scheduling related data being stored in spreadsheet files. Therefore an initial conclusion can be made that the data adapter would be required to have the ability to capture data stored in an ERP system and also to capture data stored in such spreadsheet based files.

Company 3 is a manufacturing SME in the healthcare and automotive sector, with between 51 and 100 employees. They have two plants with multiple lines, high changeover times and in general their actual demand is aligned with their forecasts. They require DES to aid in the understanding of how to increase their machine utilization and sequence dependency of their changeovers, however while some of their data is stored electronically they have a poor visibility of data throughout their system. They also have a stock management system in place. In this case, due to poor visibility of data, elicited requirements derived include providing the ability to aid in improving their understanding of where data relevant to the problem being simulated is stored. The tool would also have to have the ability to capture paper based and electronically stored data from various systems.

Company 4 is a manufacturing SME in the food sector with between 101 and 200 employees. They have approximately 6 different sequence dependent product lines with a number of different variables in these lines. They require a simulated solution in order to reduce the effect of bottlenecks in their systems. 


\section{Byrne, Byrne, Carvalho e Ferreira, and Ivers}

Their data is stored electronically alongside paper-based scheduling, therefore the tool should have the ability to integrate with both electronic data and capture data that is stored in paper format.

Table 1: Preliminary SME DES data case studies (manufacturing SMEs)

\begin{tabular}{|l|l|l|l|l|l|}
\hline Company & Sector & Size & $\begin{array}{l}\text { Production Pro- } \\
\text { cess }\end{array}$ & Data & $\begin{array}{l}\text { High-level Elic- } \\
\text { ited Require- } \\
\text { ments }\end{array}$ \\
\hline 1 & Food & $\begin{array}{l}{[51-100]} \\
\text { Employees }\end{array}$ & $\begin{array}{l}\text { Manufacture of food } \\
\text { products. Typically } \\
\text { low volumes, high } \\
\text { variety. Orders arrive } \\
\text { daily and forecasts } \\
\text { have 5-10\% variance }\end{array}$ & $\begin{array}{l}\text { Manual paper } \\
\text { based data record- } \\
\text { ing, and account- } \\
\text { ing software not } \\
\text { used extensively. }\end{array}$ & $\begin{array}{l}\text { Ability to capture } \\
\text { manual paper- } \\
\text { based data, } \\
\text { Ability to capture } \\
\text { human-based sys- } \\
\text { tems knowledge }\end{array}$ \\
\hline 2 & Medical & $\begin{array}{l}{[201-300]} \\
\text { Employees }\end{array}$ & $\begin{array}{l}\text { Highly automated, } \\
\text { unscheduled down- } \\
\text { time of machines and } \\
\text { changeovers can be } \\
\text { significant. Changeo- } \\
\text { ver time is sequence } \\
\text { dependent }\end{array}$ & $\begin{array}{l}\text { ERP system to } \\
\text { store system and } \\
\text { process data, } \\
\text { Spreadsheet for } \\
\text { scheduling }\end{array}$ & $\begin{array}{l}\text { Ability to capture } \\
\text { data from database } \\
\text { systems and } \\
\text { spreadsheet based } \\
\text { data }\end{array}$ \\
\hline 3 & $\begin{array}{l}\text { Healthcare } \\
\text { and Auto- } \\
\text { motive }\end{array}$ & $\begin{array}{l}{[51-100]} \\
\text { Employees }\end{array}$ & $\begin{array}{l}\text { Two plants, multiple } \\
\text { lines, high changeo- } \\
\text { ver times, demand } \\
\text { aligned with forecasts }\end{array}$ & $\begin{array}{l}\text { Poor visibility of } \\
\text { data, some stored } \\
\text { electronically, } \\
\text { some paper based. } \\
\text { Stock management } \\
\text { system. }\end{array}$ & $\begin{array}{l}\text { Ability to capture } \\
\text { paper-based and } \\
\text { electronically } \\
\text { stored data from } \\
\text { various systems }\end{array}$ \\
\hline 4 & Food & $\begin{array}{l}{[101-200]} \\
\text { Employees }\end{array}$ & $\begin{array}{l}\text { Approximately 6 } \\
\text { product lines, se- } \\
\text { quence dependent }\end{array}$ & $\begin{array}{l}\text { Electronic storage } \\
\text { of data alongside } \\
\text { manual scheduling } \\
\text { (paper based) }\end{array}$ & $\begin{array}{l}\text { Ability to capture } \\
\text { both paper-based } \\
\text { and electronically } \\
\text { stored data }\end{array}$ \\
\hline
\end{tabular}

Based both on requirements elicited during interaction with companies and with additional requirements from focused requirements sessions, Table 2 gives an initial list of high-level base requirements for the SME data adapter. Note that this list is not exhaustive. The first requirement is one derived across companies studied, namely the ability to capture paper-based DES-relevant data, such as hard-copy files stored related to a process. Following this, the second requirement is the ability to capture data relevant to the DES model stored in database based systems, such as data persisted by customer relationship management or ERP systems. The tool needs to have the capability to capture knowledge stored by humans, and this is the third requirement. The fourth requirement is the ability to capture data stored in files, such as spreadsheets that are relevant to the DES model being developed. The capability to interoperate with Cloud based systems is an important requirement, and this fifth requirement includes such systems as ERP systems specifically designed for SMEs hosted in Cloud environments. The sixth requirement is for a form of templating system, to allow users to select from the type of problem faced or the type of DES model to be built to solve specific problems for which data is required. This is supported by the seventh requirement which is for a mechanism to relay expert information relating to the location and type of data required by the DES model based on the template selected with support for interacting with the data. The eighth requirement is for the ability to capture information relating to the solutions provided when relaying expert information. The last functional requirement on the list is the ninth requirement, which is is for the ability for the data to be input to the data adapter tool where data is not readily available within SMEs. In this case, the DES modeler (or other) would be required to understand the context or type of data necessary, and should have support from the tool for understanding what data is missing or required by the DES model that is not readily available. Additionally, there are two non-functional requirements. The first 
relates to security, as there is a need for a high level of security with the potential use of sensitive company data in order to enable a high level of trust in the use of the tool. The second non-functional requirement relates to the cost of deploying and operating the data adapter, which should be low enough to be suitable for use by SMEs. Taking these requirements into account, the following section details a proposed SME data adapter architecture.

Table 2: Initial Base High-Level Requirements List for SME Data Adapter

\begin{tabular}{|l|l|l|}
\hline $\begin{array}{l}\text { Requirement } \\
\text { Number }\end{array}$ & Requirement Type & Requirement \\
\hline 1 & Functional & Ability to capture "paper-based" DES-relevant data \\
\hline 2 & Functional & $\begin{array}{l}\text { Ability to capture DES-relevant data stored in database-based systems } \\
\text { (e.g. MES, ERP, CRM, SRM) }\end{array}$ \\
\hline 3 & Functional & Ability to capture "human-based" and tacit systems knowledge \\
\hline 4 & Functional & $\begin{array}{l}\text { Ability to capture DES-relevant data stored in files (e.g. Microsoft Ex- } \\
\text { cel) }\end{array}$ \\
\hline 5 & Functional & $\begin{array}{l}\text { Interoperable with Cloud based systems which store SME DES-relevant } \\
\text { data (e.g. SAP Business By Design) }\end{array}$ \\
\hline 6 & Functional & Templating system to select problem scenarios \\
\hline 7 & Functional & $\begin{array}{l}\text { Mechanism to relay suggested data related solutions based on template } \\
\text { with support }\end{array}$ \\
\hline 8 & Functional & $\begin{array}{l}\text { Ability to capture information relating to the solutions from require- } \\
\text { ment 7 }\end{array}$ \\
\hline 9 & Functional & Ability to input data where relevant data is not available \\
\hline 10 & Non-functional & High level of Security \\
\hline 11 & Non-functional & Low-cost deployment and operational model \\
\hline
\end{tabular}

\section{SME DATA ADAPTER DESIGN}

Taking the requirements presented in Section 3 into consideration, this section provides both design decisions made in order to meet the specified requirements, and an architectural design for a proposed SME data adapter.

\subsection{Design Decisions to Meet Requirements}

An overview of the initial design decisions made to meet the SME data adapter requirements given in Table 2 is presented in Table 3. Referring to Table 3, the requirement for the ability to capture paper-based DES-relevant data is met through the provision of a templating system and graphical user interface (GUI) which enables users to input data from paper-based sources. The templating system in place would aid in suggesting the type of data required by the DES model from these sources. The second requirement for capturing DES relevant data stored on-premise is met through the proposed implementation of a connectivity agent with capability to connect to the systems the data is stored in (such as ERP or CRM systems). Examples of the types of connectivity functionality required would include open database connectivity (ODBC) and object linking and embedding database (OLE-DB) capabilities.

An important requirement to be met is the capability to capture human-based systems knowledge, as a lot of knowledge and expertise relating to data required by a DES model can reside in humans. Therefore a potential design decision to meet this requirement would be the use of an expert system with an input mechanism such as a knowledge acquisition facility to capture this knowledge. A knowledge-base in the form of a database would be used to persist this knowledge for future use by the expert system. A templating system and purpose-built GUI would also support this.

In terms of the fourth requirement, through capturing DES relevant data stored locally in files a local connectivity agent could be used to support this with the capability to read from a number of different file types, as well as the capability to add customized scripts where new file types need to be read from. 


\section{Byrne, Byrne, Carvalho e Ferreira, and Ivers}

Again, a database would be used to store the results of this exercise. Regarding the ability to interoperate with Cloud based systems which may be used to store SME-relevant data, networking capability and a server-side connectivity agent would potentially be used. Once this information is pulled from the remote system, the data could be persisted in the data adapter in a database. The sixth requirement refers to a templating system to select problem scenarios under consideration by the DES model, and a templating system could be deployed to support this, which would be configurable and customizable.

Table 3: Initial design decisions to meet SME data adapter requirements

\begin{tabular}{|c|c|}
\hline $\begin{array}{l}\text { Requirement } \\
\text { Number }\end{array}$ & Proposed Architectural Design Decision \\
\hline 1 & $\begin{array}{l}\text { Templating system and GUI to enable input of DES relevant paper-based } \\
\text { data. Database to persist data for later use. }\end{array}$ \\
\hline 2 & $\begin{array}{l}\text { Client-side connectivity agent with connectivity capability (e.g. ODBC, } \\
\text { OLE DB, JDBC, etc.) and administration GUI to support connection set- } \\
\text { up }\end{array}$ \\
\hline 3 & $\begin{array}{l}\text { Templating system, GUI and Expert system to aid in understanding what } \\
\text { data is required to be captured by a human with input mechanism and } \\
\text { ability to persist of information (database) }\end{array}$ \\
\hline 4 & $\begin{array}{l}\text { Client-side connectivity agent with connectivity capability to read from a } \\
\text { number of file types. Additional possibility to add customised scripts. } \\
\text { Database to persist data gathered for use. }\end{array}$ \\
\hline 5 & $\begin{array}{l}\text { Networking capability and server-side connectivity agent to enable read- } \\
\text { ing from Cloud based systems. }\end{array}$ \\
\hline 6 & $\begin{array}{l}\text { Customisable templating system enabling selection of high level SME } \\
\text { scenarios. }\end{array}$ \\
\hline 7 & $\begin{array}{l}\text { Expert system with explanation facility to relay information and suggest } \\
\text { possible DES data-related solutions. Stored in knowledge base in a data- } \\
\text { base. }\end{array}$ \\
\hline 8 & Expert system with acquisition facility for capturing knowledge. \\
\hline 9 & $\begin{array}{l}\text { Templating system and input facility through GUI, persisted in a data- } \\
\text { base and supported by an expert system }\end{array}$ \\
\hline 10 & $\begin{array}{l}\text { Highly secure technological implementation with high level of authenti- } \\
\text { cation and session management }\end{array}$ \\
\hline 11 & $\begin{array}{l}\text { Cloud deployment to enable low cost deployment and low price setting } \\
\text { for SMEs }\end{array}$ \\
\hline
\end{tabular}

In providing the ability to relay capture information relating to solutions from the template, an expert system with an explanation facility could be provided which would suggest possible DES-related data solutions. The knowledge associated with the expert system would be stored in its own knowledge base in the form of a database. The ninth requirement refers to the capability to input data where data required by the DES model from the SME for the problem to be simulated is not available. This can be solved by providing for this capability in the templating system supported by the expert system in explaining what data is necessary and providing an input mechanism through a GUI to enter this data into the system, which would be persisted in a database.

A high level of security would need to be provided with high levels of authentication and session management in order for trust to be inherent in the vendor, and a low cost deployment and operational model would be required in order for it to be priced low enough to be suitable for use by SMEs. In this case, providing a Cloud based solution would be of benefit as it would allow for an on-demand pricing model so that the option would be available for the SME to only pay for the system as they require it. The vendor in this case would also benefit through the ability to operate the solution as a service at lower cost. 


\section{Byrne, Byrne, Carvalho e Ferreira, and Ivers}

\subsection{Architectural Design}

As can be seen in Figures 1 and 2, a design solution and component diagram with sub-component dependencies are presented respectively whereby the SME data adapter application is deployed on the Cloud for use. There are three deployment approaches realized by this design, namely on-premise, ondevice and on-demand, as follows:

- On-Premise: On-premise covers anything that exists within the walls of the SME, whether this is internally on private Cloud, on local servers with client access, on client, or (in the case of data) in hard-copy format or knowledge not formally recorded (in people).

- On-Device: On-device covers any mobile device used internally or externally to the SME capable of connecting to a network and with an operating system supporting Web browsers or Web-capable applications.

- On-Demand: On-demand includes any part of the SME data adapter that is deployed (in this case) in the Cloud and can be accessed through a software-as-a-service (SaaS) deployment model.

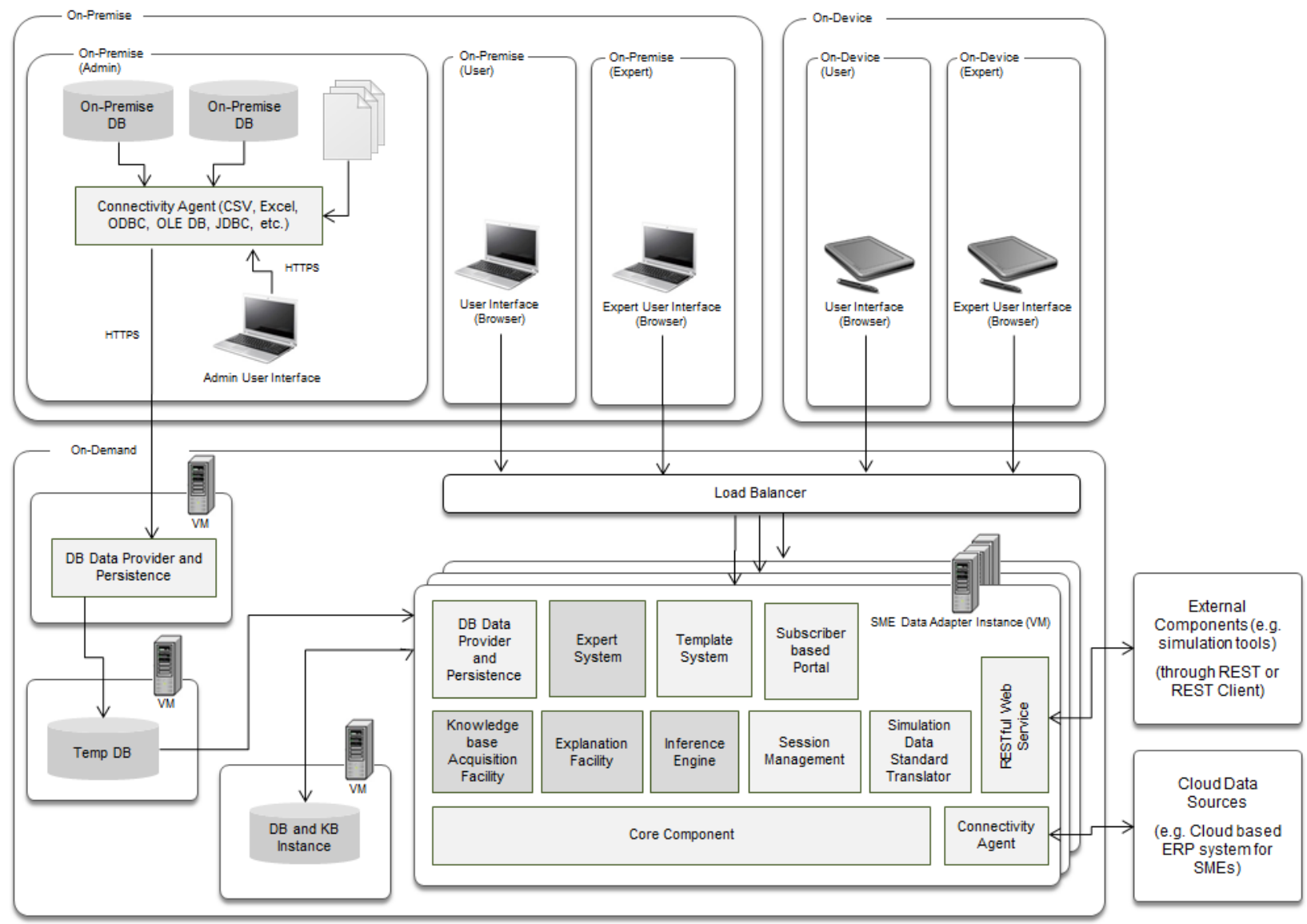

Figure 1: Architectural design for Cloud-based SME data adapter

Referring to Figure 1, the on-premise deployment approach contains a connectivity agent which can be used to connect to and read from data sources. These data sources include both on-premise databases, interacting with system APIs, and locally stored files. The administrator user interface is used to administrate the connections on-site with the data sources, once access is supplied by the SME. The required data is persisted remotely in a database instance on the Cloud pushed over a secure connection which can then be read from the SME data adapter instance. In addition, there are browser based graphical user interfaces provided on-premise (or client side external to the SME) used by both a user of the SME data adapter, or 
an expert interacting with the expert system on the Cloud contained within the SME data adapter. Note that the general vision for an SME data adapter presented here can equally be deployed as a full private Cloud on-premise solution.

The on-device approach includes additional user interfaces for both users and experts to use mobile solutions to access the data adapter extending to mobile devices such as iPads, smartphones and other such devices. An example of a user in this case would be a DES modeler who needs to be supported in both understanding what data is relevant to the system being modeled, where to locate the data, how to obtain the data and supported in physically making the relevant connections to read the data into the adapter. Their primary interaction with this support is through the user interfaces. The expert in this case can add expertise to the system through the user interface in order to improve and update the data-related knowledge stored in the system.

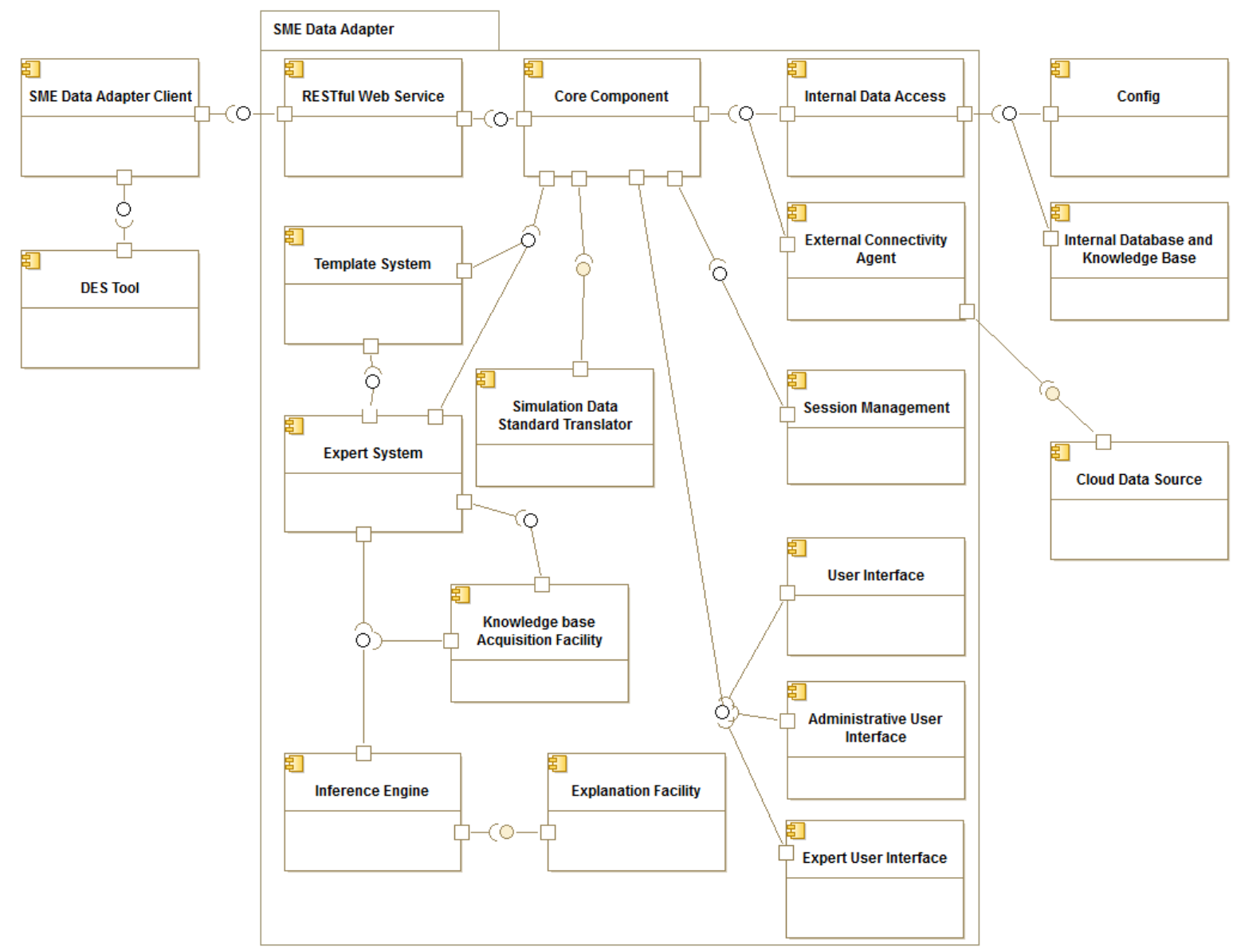

Figure 2: Component-based architectural design for Cloud-based SME data adapter

The on-demand approach includes a database instance, a temporary database with data provider and persistence component, a load balancer and a number of SME data adapter instances (a component architectural diagram for which is also given in Figure 2). The database is used to store all data relevant to the SME data adapter, for example all user information, log in credentials, session information, data relating to templates, translation of data to a standard format and all data stored relevant to the specific DES cases. In addition it contains the full knowledge base used by the expert system. The database data provider is used to temporarily take the data pushed from the client side and persist it in a temporary database, which can be read by the data adapter. The load balancer is used to balance the load across instances of the SME data adapter. 
Focusing on the SME data adapter component (see both Figures 1 and 2), it exists as a VM instance and has a number of sub-components. These sub components include the core component, a data provider, a template system, a RESTful Web service API, session management, a Simulation Data Standard translator, and an expert system with a knowledge base acquisition factility, inference engine and explanation facility.

The core sub component contains the processing logic in the system and supports all interaction between other sub components in the data adapter component. The data provider is the main component interacting between the data adapter and databases. It can create, read, update and delete to/from the temporary database (which stores the data that has been pushed from on-premise), the main database and the knowledge base (which is contained within the main database).

The template system provides a mechanism by which templates for core manufacturing cases can be selected, and a pre-defined template for each can be created which describes the data required by the system. New templates can be added or customized as required by users, and an additional proposed benefit is that these templates can be submitted to the vendor through the subscriber portal to be available to all users of the data adapter. Using these templates, data can be generated where none is available in an SME, including data related to processes and procedures.

Session management is necessary in order to keep connections open with clients during a session whereby they are logged into the adapter. The state and page information is maintained over multiple requests for the same or different pages in the adapter.

The expert system supports the templating system and is in place to capture and preserve irreplaceable human expertise as to where to access data. It provides a way to solve the problem of where and how to gather the data, and is more consistent than human experts would be in this space. In addition, because it is deployed on the Cloud, experts across a wide range of SMEs can add their knowledge to the expert system and make this public to be shared with others, thus making the knowledge more extensive and correct. The expert system is comprised of a number of inter-related sub components, these being the knowledge base, the knowledge base acquisition facility, the inference engine, the explanation facility and the user interface.

The knowledge base is stored in the general database component instance and accessed through the core component by the inference engine. It stores all relevant information, data, rules, cases and relationships that the expert system uses in relation to the knowledge of the different problem scenarios to be simulated by SMEs. These scenarios, otherwise known as cases, are used in developing solutions to the problems to be simulated. The process involves finding cases stored in the knowledge base that are similar to the scenario to be modeled and modifying the solution in terms of the data required for the cases to fit or accommodate the scenario. Over time, a large amount of information relevant to a specific problem scenario (case) can be built up through the template, that is either specific to one SME or generic across SMEs for specific problem scenarios.

The inference engine is used to seek information from the knowledge base and to provide answers, predictions and suggestions in a similar way that a human expert would. Forward chaining is used in this case, whereby the facts are started with and worked forward to the conclusion as to what data is required and where to potentially locate it. The explanation facility is used to enable a user or decision maker to understand how the expert system arrived at certain conclusions or results in determining the data required by the DES scenario, the potential location of this data and how it can be interacted with. In this way, the facts and rules that were used in reaching the conclusion can be indicated to the user.

Creating and updating the knowledge base is an on-going process. When the tool is initially deployed, the knowledge base is populated with the vendor's understanding of the generic scenarios faced by SMEs when attempting to develop DES models, and a mechanism to allow for data that isn't available to be "filled" based on assumptions. However the tool provides a knowledge acquisition facility to provide a convenient and efficient means to capture and store all components of the knowledge base related to specific template SME cases.

The knowledge acquisition facility provides both manual and automated procedures for populating the knowledge base which must be updated and validated regularly. This is accessed through the graph- 


\section{Byrne, Byrne, Carvalho e Ferreira, and Ivers}

ical user interface either on-premise or on-device. Using knowledge from multiple SMEs, the vendor can harvest DES-related information in order to enhance the general templates and improve the available knowledge. The user can choose whether to make the knowledge provided to the tool either private or public.

This general subscriber-based user portal acts as a mechanism by which DES modelers can communicate and give feedback on the SME templates provided and provides a forum by which DES modelers can support each other in understanding how to access DES-related data. This gives the added benefit of providing requirements to the vendor for future releases of the tool.

The DES data standard translator translates the data required into a standard format for use by the a DES tool(s). This could potentially be in CMSD format, SysML format or other. The representational state transfer (REST)ful Web service provides a Web API and associated downloadable client in order to enable interoperability with the system from an external source. This enables (for example) an external DES tool to read the data contained in the SME data adapter whether it is in CMSD format or other.

Finally, a customizable connectivity agent gives the ability to connect to Cloud (or networked) data sources which store SME data. An example of this would be a Cloud based ERP system such as SAP Business By Design or NetSuite ERP.

\section{CONCLUSIONS AND FUTURE WORK}

Discrete event simulation (DES) is a technique used extensively and effectively by large companies to optimize their processes. However, DES is not widely used by small to medium sized enterprises (SMEs) due to complexity and cost being prohibitively high. In SMEs, the data gathering and preparation phase is where complexity and effort required are highest when developing a simulation model, leading to high costs in order to avoid the potential for erroneous results due to incorrect assumed or real input data.

This work specifically addresses this phase with a view to reducing this complexity and effort. The proposed solution is a software adapter that connects to existing data sources and/or fills data gaps using a data generator, including processes and procedures for implementation scenarios. The resulting Cloud based solution enables SMEs to take advantage of the benefits of DES with reduced complexity leading to reduced cost and higher result accuracy and validity. The RESTful API gives external components or tools the ability to easily integrate or interoperate with the data adapter. Deploying such a solution on the Cloud has a number of advantages. Information can be built up on multiple cases over time and the knowledge base can grow relating to generic data requirements faced by SMEs for specific problem scenarios. This allows expertise to come from those associated with the problem domain, and gives the ability for the simulation modelers using this tool to share expert knowledge across domains and across SME types and problem scenario types relating to the data that is required for a simulation model, under control of the vendor. In addition, the tool can be set at a lower price point to the SME or the simulation modeler employed by the SME due to the vendor paying for infrastructure used on-demand, aiding in reducing the overall cost of a simulation effort to the SME. Also it gives the potential for increased interoperability with other SME focused Cloud based solutions on the market. Additionally it enables the data adapter to scale up or down in response to increased or decreased demand for use of the tool by SMEs or simulation modelers leading to decreased vendor TCO.

Future work includes elaborating on the proposed solution, expanding the requirements through more detailed requirements elicitation and analysis (both qualitative and quantitative), implementation of the solution and field testing of the proposed system across multiple SMEs and problem scenarios. In addition, there is potential for future features to be investigated, including adding data analysis capability (through distribution fitting or other) through further development or integration with existing tools on the market. 
Byrne, Byrne, Carvalho e Ferreira, and Ivers

\section{ACKNOWLEDGMENTS}

This work was conducted by Dublin City University and is funded by Enterprise Ireland under project reference number CF-2012-2647. See http://www.sim.ie

\section{REFERENCES}

Balci, O. 1994. "Validation, verification, and testing techniques throughout the life cycle of a simulation study." In Proceedings of the 1994 Winter Simulation Conference, Edited by J. D. Tew, S. Manivannan, D. A. Sadowski, and A. F. Seila, 215-220. Piscataway, New Jersey: Institute of Electrical and Electronics Engineers, Inc.

Balci, O. 1997. "Verification, validation and accreditation of simulation models." In Proceedings of the 1994 Winter Simulation Conference, Edited by S. Andradottir, K. J. Healy, D. H. Withers, and B. L. Nelson, 135-141. Piscataway, New Jersey: Institute of Electrical and Electronics Engineers, Inc

Bengtsson, N., G. Shao, B. Johansson, Y. T. Lee, A. Skoogh, and C. Mclean. 2009. "Input Data Management Methodology for Discrete Event Simulation." In Proceedings of the 2009 Winter Simulation Conference, Edited by M. D. Rossetti, R. R. Hill, B. Johansson, A. Dunkin, \& R. G. Ingalls, 1335-1344. Piscataway, New Jersey: Institute of Electrical and Electronics Engineers, Inc.

Bergmann, S., S. Stelzer, and S. Straßburger, 2011. "Initilization of Simulation Models using CMSD." In Proceedings of the 2011 Winter Simulation Conference, Edited by S. Jain, R. Creasey, J. Himmelspach, K. P. White, and M. C. Fu. 2228-2239. Piscataway, New Jersey: Institute of Electrical and Electronics Engineers, Inc.

Casson, M. 1982. The entrepreneur: An economic Theory. NJ: Barnes \& Noble Books.

European Commission. 2003. "Small and medium-sized enterprises." Accessed July 11, 2013, http://ec.europa.eu/enterprise/policies/sme/facts-figures-analysis/sme-definition/index_en.htm.

Huang, E., R. Ramamurthy, and L. F. McGinnis. 2007. "System and Simulation Modelling Using SYSML." In Proceedings of the 2007 Winter Simulation Conference, Edited by S. G. Henderson, B. Biller, M.-H. Hsieh, J. Shortle, J. D. Tew, \& R. R. Barton. 796-803. Piscataway, New Jersey: Institute of Electrical and Electronics Engineers, Inc.

Koh, S. C. L., and S. M. Saad. 2002. "Development of a business model for diagnosing uncertainty in ERP environments." International Journal of Production Research, 40(13): 3015-3039.

Law, A. M. 2009. "How to build valid and credible simulation models." In Proceedings of the 2009 Winter Simulation Conference, Edited by M. D. Rosetti, R. R. Hill, B. Johansson, A. Dunkin, \& R. G. Ingalls. 24-33. Piscataway, New Jersey: Institute of Electrical and Electronics Engineers, Inc.

Leong, S., Y. T. Lee, and F. Riddick. 2006. "A Core Manufacturing Simulation Data Information Model for Manufacturing Applications." In Proceedings of the Systems Interoperability Standards Organization 2006 Fall Simulation Interoperability Workshop. 1-7.

Liston, P., E. Kabak, P. Dungan, J. Byrne, P. Young, and C. Heavey. 2010. "An Evaluation of SysML to support Simulation Modeling." In Conceptual Modelling for Discrete Event Simulation, Edited by S. Robinson, R. Brooks, K. Kotiadis, and D-J. Van Der Zee. CRC Press

O'Kane, J., A. Papadoukakis, and D. Hunter. 2007. "Simulation usage in SMEs." Journal of Small Business and Enterprise Development, 14(3): 514-527.

Riddick, F., and Y. T. Lee. 2008. "Representing Layout Information in the CMSD Specification." In Proceedings of the 2008 Winter Simulation Conference, Edited by S. J. Mason, R. R. Hill, L. Mönch, O. Rose, T. Jefferson, \& J. W. Fowler. 1777-1784. Piscataway, New Jersey: Institute of Electrical and Electronics Engineers, Inc.

Simchi-Levi, D., P. Kaminsky, and E. Simchi-Levi. 2003. "Designing and Managing the Supply Chain: Concepts, Strategies and Case Studies" (2nd edition). New York: McGraw Hill.

U.S. Small Business Administration. 2013. "Table of Small Business Size Standards." Accessed July 11, 2013, http://www.sba.gov/sites/default/files/files/Size_Standards_Table_updated 070213(2).pdf 
Byrne, Byrne, Carvalho e Ferreira, and Ivers

\section{AUTHOR BIOGRAPHIES}

JAMES BYRNE is a Senior Researcher in the DCU Business School at Dublin City University. Prior to this he worked as a Specialist Researcher for SAP Research, and as a Postdoctoral Researcher in the University of Limerick. His research interests include Web and Cloud based simulation, supply chain management, discrete event simulation, Cloud computing, virtualization and cost and risk related advanced analytical techniques. He holds a B.Tech in Information Technology and Telecommunications, an M.Tech in Computer Integrated Manufacturing and a Ph.D from the Manufacturing and Operations Engineering department in the University of Limerick. His Ph.D is in the area of Web based simulation applied to contract costing for outsourcing enterprises. He is a member of IEEE and IET. His email address is james.byrne@dcu.ie.

PJ BYRNE is Head of the Management Group and Senior Lecturer at Dublin City University Business School. His main teaching focus is in the areas of supply chain management and business process improvement. He holds a B.Eng in Production Engineering and a PhD from the Manufacturing and Operations Engineering department in the University of Limerick. His PhD is in the area of large scale industrial supply chain simulation modeling. His research interests include supply chain design, analysis and optimization, cloud based simulation, discrete event simulation, industrial applications of simulation modeling for the manufacturing and services sectors and business process optimization. Dr. Byrne has an extensive track record in industrial based research and is currently a Principal Investigator in three externally funded research projects. To date, he has been successful, with his research team in attracting $€ 1.5$ million in research funds, primarily in the area of discrete event simulation. This funding has been obtained through EU framework programs, national funding and industrial sponsorship. His email address is pj.byrne@dcu.ie

DIANA CARVALHO E FERREIRA is a Postdoctoral Researcher in the DCU Business School at Dublin City University. After completing her BSc and MSc in Informatics Engineering at the University of Coimbra, with First Class Honors, Diana joined the MIT-Portugal Program to pursue her Ph.D at Instituto Superior Tecnico, in Lisbon. She was awarded a Visiting Ph.D Studentship at MIT and a 4 year Doctoral Research Grant from the Portuguese Foundation for Science and Technology. Her email address is diana.ferreira@dcu.ie.

ANNE MARIE IVERS is a Postdoctoral Researcher in the DCU Business School at Dublin City University. She holds a B.BS in Marketing, an M.BS in Marketing and a Ph.D from Waterford Institute of Technology. Her research interests are primarily in the field of marketing capabilities, business networks, business strategy, simulation modeling and management. Her email address is anne-marie.ivers@dcu.ie. 